\title{
Theory of the Isotropic-Nematic-Nematic Phase Separation for a Solution of Bidisperse Rodlike Particles
}

\author{
G. J. Vroege* and H. N. W. Lekkerkerker" \\ Van't Hoff Laboratory, University of Utrecht, Padualaan 8, 3584 CH Utrecht, The Netherlands
}

Received: September 25, 1992

\begin{abstract}
Nematic-nematic phase equilibria and an isotropic-nematic-nematic triple point are located in the phase diagram of a bidisperse solution of rigid hard rods. To this end, the formulation of the Onsager second virial theory for bidisperse systems using Gaussian trial functions is applied. It appears that-within this approximation-the nematic-nematic transition is not caused by excluded-volume effects but by a balance between orientational entropy and entropy of mixing. Below a length ratio of 3.167 there is only a simple isotropic-nematic transition present.
\end{abstract}

\section{Introduction}

In the 1940 s Onsager presented ${ }^{2}$ his theory of the isotropicnematic transition of a monodisperse system of thin hard rods, which was published in his seminal paper of 1949.1 Onsager showed that a phase transition can be explained on the basis of merely two-particle interactions represented by the second virial term in an expansion of the free energy of the system. The volume fraction at which the transition appears was predicted to be inversely proportional to the aspect ratio of the rods, which leads to an exact limit of the Onsager theory for infinitely thin rods. Despite its great theoretical importance, the direct relevance of the Onsager theory for the explanation of experiments is rather limited being restricted to low volume fractions of perfectly rigid rods. However, its extensions obtained by introducing semiflexibility and developments for higher volume fractions have been applied with good results to polymer and colloidal solutions. (See, e.g., reviews ${ }^{3-8}$ and references quoted there.)

Another factor, which is of importance for the interpretation of experimental results, is the influence of polydispersity. Onsager already hinted at this in his original paper' and even devoted an appendix to calculating the required integrals in bidisperse systems for his choice of the orientational distribution functions. In the context of the Onsager theory, the first numerical results for bidisperse systems were obtained by Lekkerkerker et al., ${ }^{9}$ who attacked this problem with numerical calculations based on series expansions of the orientational distribution function. They found several interesting results like a strong fractionation effect (with the longer rods going preferentially to the nematic phase), a widening of the biphasic gap, and a reentrant phase phenomenon. A few years ago, Birshtein et al. ${ }^{18}$ also located a triphasic and nematic-nematic area in a bidisperse system with length ratio 5 applying the method of Lekkerkerker et al.,9 which were not detected in the original paper.

Triphasic equilibria had emerged previously in calculations on bidisperse rodlike systems by Flory and Abe, " based on the lattice theory developed by Flory. ${ }^{12}$ Although the Flory theory does not reduce to the exact limit of the Onsager theory for infinitely thin rods, it generally shows the same qualitative trends. (For reviews on the Flory theory, see e.g. refs 13 and 14.) Experimentally, triphasic equilibria were also observed, e.g., in bidisperse systems of schizophyllan in water ${ }^{15,16}$ and bimodal solutions of imogolite. ${ }^{17}$

The above-mentioned calculations of triphasic phenomena are all based upon numerical procedures and consequently tend to obscure the physical mechanism involved. For this reason an analytical theory may be helpful for a better understanding. Indeed, analytical calculations by Odijk and Lekkerkerker, ${ }^{10}$ based on a simplified version of Onsager's orientational distribution, the so-called Gaussian distribution function, successfully explained results like the fractionation effect, the widened biphasic gap, and a reentrant nematic-isotropic transition. In this paper we show that triphasic and nematic-nematic equilibria may also be located within the Gaussian approach. Remarkably, the underlying mechanism for the nematic-nematic phase transition does not involve changes in excluded volume but a balance between orientational entropy and entropy of mixing.

\section{Starting Equations}

Our discussion of nematic-nematic phase equilibria and the triphasic isotropic-nematic-nematic equilibrium in bidisperse rodlike systems is entirely based upon equations derived in the paper by Odijk and Lekkerkerker. ${ }^{10}$ We will not repeat the derivation of these equations in detail but summarize the pertinent equations in this section and discuss them along the way. To facilitate comparison with ref 10 we adhere to its notation as much as possible.

We start with a solution of hard rods with the same diameter $D$ but two different lengths $L_{j}(j=1,2$; for sake of definiteness we denote the longer rods by subscript 2 ). The volume of the system is $V$, and the number of short and long rods is $N_{1}$ and $N_{2}$. Onsager ${ }^{i}$ described the nematic phase of a dilute solution of long rods in terms of function $f_{j}(\theta)$ which describes the distribution of angles $\theta$ of the rods of type $j$ around the director. The occurrence of an isotropic state (with $f_{j}$ constant) or a nematic state (with a peaked distribution) is caused by a competition between orientational entropy and translational entropy. Onsager defined $\sigma_{j}$ as a measure for the orientational entropy

$$
\sigma_{j} \equiv \int f_{j}(\theta) \ln \left[4 \pi f_{j}(\theta)\right] \mathrm{d} \Omega
$$

which acquires its minimum value of 0 in the isotropic state but increases as the orientational entropy decreases. $d \Omega$ indicates integration over all angles. The relevant part of the translational entropy derives from the two-particle interaction in the form of the excluded volume between two hard rods (with length $L_{j}$ and $L_{k}$ ) which depends on their mutual angle $\gamma$

$$
v_{\text {excl }} \sim 2 D L_{j} L_{k}|\sin \gamma|
$$

A measure for the average excluded-volume interaction between rods of type $j$ and $k$ is given by the average of its angular dependence

$$
\rho_{j k} \equiv(4 / \pi) \iint|\sin \gamma| f_{j}(\theta) f_{k}\left(\theta^{\prime}\right) \mathrm{d} \Omega \mathrm{d} \Omega^{\prime}
$$

which is 1 in the isotropic state and more favorable (between 0 and 1) in the nematic state. The total free energy $F$ of the 
bidisperse system may be written in terms of these parameters as

$$
\begin{aligned}
& \frac{F}{\left(N_{1}+N_{2}\right) k_{\mathrm{B}} T} \simeq \\
& \mathrm{cst}+ \ln c^{-1}+(1-x) \ln (1-x)+x \ln x+(1-x) \sigma_{1}+ \\
& x \sigma_{2}+c\left[(1-x)^{2} \rho_{11}+2 x(1-x) q \rho_{12}+x^{2} q^{2} \rho_{22}\right]
\end{aligned}
$$

where $k_{\mathrm{B}}$ and $T$ have their usual meaning of Boltzmann's constant and absolute temperature. In this equation $x \equiv N_{2} /\left(N_{1}+N_{2}\right)$ is the mole fraction of the longer rods, and $c$ is the total number density of rods rendered dimensionless by relating it to the excluded volume $b$ between two randomly oriented short rods

$$
c \equiv b \frac{N_{1}+N_{2}}{V} \equiv \frac{\pi}{4} L_{1}^{2} D \frac{N_{1}+N_{2}}{V}
$$

Since $c$ is related to the excluded volume of two short rods, the excluded-volume terms in eq 4 which involve one or two longer rods are multiplied by $q$ or $q^{2}$, with

$$
q \equiv L_{2} / L_{1}
$$

Once expression 4 for the free energy is known, we must minimize it with respect to the orientational distribution at a given $c$ and $x$. One way of doing this is using a trial function with one or more variational parameters. A convenient choice for the angular distribution of rod $j$ in the nematic state is the Gaussian trial function 5.10

$$
\begin{aligned}
f_{j}(\theta) & \equiv \frac{\alpha_{j}}{4 \pi} \exp \left(-\frac{1}{2} \alpha_{j} \theta^{2}\right) \quad 0 \leq \theta \leq \pi / 2 \\
& \equiv \frac{\alpha_{j}}{4 \pi} \exp \left(-\frac{1}{2} \alpha_{j}(\pi-\theta)^{2}\right) \quad \pi / 2 \leq \theta \leq \pi
\end{aligned}
$$

which gives good qualitative results for the isotropic-nematic phase transition for monodisperse rods and is getting more accurate as the phase is more highly ordered. $\alpha_{j}$ is the variational parameter of this trial function. To determine its value, we must know the expressions for $\sigma_{j}$ and $\rho_{j k}$ obtained by substituting eq 7 in eqs 1 and 3:

$$
\begin{gathered}
\sigma_{j} \sim \ln \alpha_{j}-1 \\
\rho_{j k} \sim \frac{4\left(\alpha_{j}+\alpha_{k}\right)^{1 / 2}}{(2 \pi)^{1 / 2} \alpha_{j}^{1 / 2} \alpha_{k}^{1 / 2}}
\end{gathered}
$$

which are the first terms of asymptotic expansions valid for large $\alpha$ s. Using these expressions in eq 4 and minimizing with respect to $\alpha_{1}$ gives us

$$
1 / 2 \pi^{1 / 2} c^{-1} \alpha_{1}^{1 / 2}=(1-x)+2^{1 / 2} x q h(Q)
$$

with definitions

$$
\begin{gathered}
Q \equiv \alpha_{2} / \alpha_{1} \\
h(Q) \equiv Q^{1 / 2} g(Q) \equiv\left(\frac{Q}{Q+1}\right)^{1 / 2}
\end{gathered}
$$

A similar equation as (10) is acquired upon minimizing $F$ with respect to $\alpha_{2}$; both equations may be combined to obtain an expression only involving the ratio of both $\alpha$ s

$$
Q^{1 / 2}=\frac{q\left[x q+2^{1 / 2} g(Q)(1-x)\right]}{\left[2^{1 / 2} h(Q) x q+(1-x)\right]}
$$

This is an implicit equation for $Q$, and although we cannot obtain an explicit form for $Q$, its value is easily found by iteration. Note that this equation only contains the mole fraction $x$ and not the concentration $c$, which implies $Q=Q(x)$. Since this in turn implies that the right-hand side of eq 10 only depends on $x$, we find that the concentration dependence of the $\alpha$ s remains quadratic like in the monodisperse case.

To locate phase transitions, we must know the osmotic pressure and chemical potential of both types of rods. These are calculated as derivatives of the free energy (4). In the isotropic phase (denoted by subscripts $i$ ) we get in dimensionless notation

$$
\begin{aligned}
& \tilde{\Pi}_{i} \equiv-\frac{b}{k_{\mathrm{B}} T}\left(\frac{\partial F}{\partial V}\right)_{N_{1}, N_{2}, T}=c_{i}+c_{i}{ }^{2}\left[\left(1-x_{i}\right)^{2}+\right. \\
& \left.\quad 2 x_{i}\left(1-x_{i}\right) q+x_{i}{ }^{2} q^{2}\right] \\
& \tilde{\mu}_{1 i} \equiv \frac{1}{k_{\mathrm{B}} T}\left(\frac{\partial F}{\partial N_{1}}\right)_{N_{2}, V, T}=\ln c_{i}+\ln \left(1-x_{i}\right)+ \\
& \quad 2 c_{i}\left[\left(1-x_{i}\right)+x_{i} q\right] \\
& \tilde{\mu}_{2 i} \equiv \frac{1}{k_{\mathrm{B}} T}\left(\frac{\partial F}{\partial N_{2}}\right)_{N_{1}, V, T}=\ln c_{i}+\ln x_{i}+ \\
& 2 c_{i}\left[\left(1-x_{i}\right) q+x_{i} q^{2}\right]
\end{aligned}
$$

where the isotropic values $\sigma_{j}=0$ and $\rho_{j k}=1$ have been used. In the nematic state (denoted by subscripts $a$ ) matters are more complicated because of expressions 8 and 9 for $\sigma_{j}$ and $\rho_{j k}$. By elaborate rearrangements ${ }^{10}$ the excluded-volume term in eq 4 could be shown equal to 2 in the Gaussian approximation, which results in simple coexistence relations

$$
\begin{gathered}
\tilde{\Pi}_{a} \sim 3 c_{a} \\
\tilde{\mu}_{1 a} \sim 3 \ln c_{a}+\ln \frac{4}{\pi}+3+\xi^{\prime}\left(x_{a}\right) \\
\tilde{\mu}_{2 a} \sim 3 \ln c_{a}+\ln \frac{4}{\pi}+3+\xi^{\prime \prime}\left(x_{a}\right)
\end{gathered}
$$

The most remarkable fact of these equations is that the osmotic pressure only depends on the total number density of rods and not on the mole fraction. The mole fraction dependence of each chemical potential is restricted to one term, which does not depend on the concentration

$$
\begin{array}{r}
\xi^{\prime}\left(x_{a}\right) \equiv \ln \left(1-x_{a}\right)+2 \ln \left(1-x_{a}+2^{1 / 2} x_{a} q h\right)- \\
\frac{2^{3 / 2}(Q-1) x_{a} g}{\left[2^{1 / 2}\left(1-x_{a}\right) g+x_{a} q\right]} \\
\xi^{\prime \prime}\left(x_{a}\right) \equiv \ln x_{a}+2 \ln \left\{q\left[2^{1 / 2}\left(1-x_{a}\right) g+x_{a} q\right]\right\}+ \\
\frac{2^{3 / 2}(Q-1)\left(1-x_{a}\right) g}{\left[2^{1 / 2}\left(1-x_{a}\right) g+x_{a} q\right]}
\end{array}
$$

By equating the osmotic pressure and chemical potentials in the isotropic and nematic phases, Odijk and Lekkerkerker ${ }^{10}$ were able to construct a fair part of the phase diagram including a reentrant phase. However, they did not notice the existence of a nematic-nematic phase transition and the corresponding isotropic-nematic-nematic triple point which we will discuss in the next sections.

\section{Coexistence between Two Nematic Phases}

When we study the possible coexistence between two nematic phases, we must realize that the osmotic pressure and chemical potentials are given by the same expressions in both phases. To obtain two different nematic phases, there must be two states, denoted by I and II, with different $c_{a}$ and/or $x_{a}$ having the same osmotic pressure and chemical potentials. When we look at eq 17 , we notice that equality of osmotic pressure requires equality 


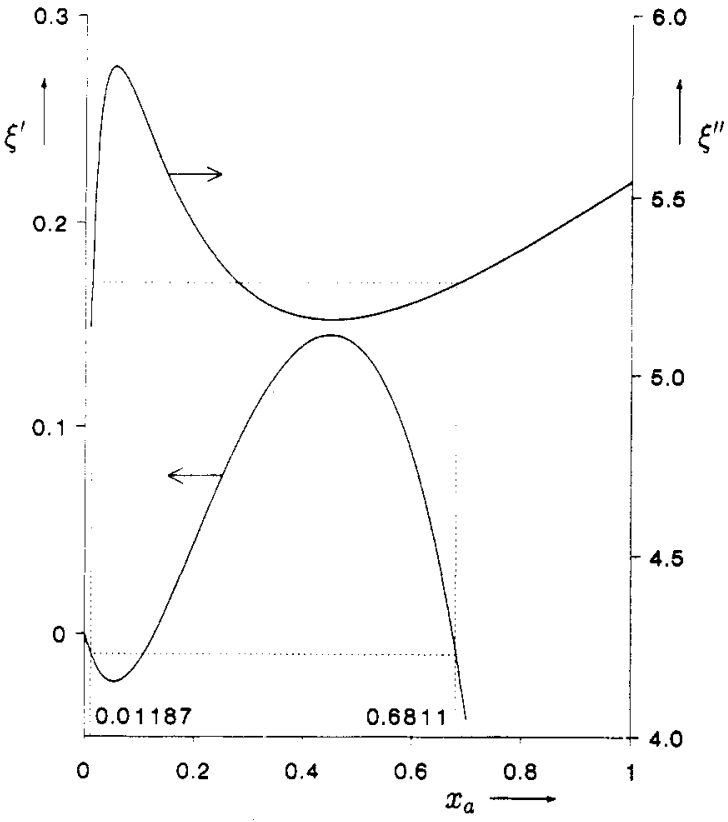

Figure 1. Mole fraction dependent parts of the chemical potentials of short and long rods for $q=4$. The dashed lines indicate the coexistence of $x_{a 1}=0.01187$ and $x_{a l l}=0.6811$, at which compositions both chemical potentials are equal.

of total number density of rods:

$$
c_{a \mathrm{l}}=c_{a \mathrm{II}}
$$

When we use this knowledge in eqs 18 and 19 , they reduce to

$$
\xi^{\prime}\left(x_{a \mid}\right)=\xi^{\prime}\left(x_{a \mid 1}\right)
$$

and

$$
\xi^{\prime \prime}\left(x_{a 1}\right)=\xi^{\prime \prime}\left(x_{a \mid 1}\right)
$$

Thus, the question of coexistence of two nematic phases is now reduced to the behavior of $\xi^{\prime}$ and $\xi^{\prime \prime}$ as a function of $x_{a}$.

In Figure 1 we give $\xi^{\prime}$ and $\xi^{\prime \prime}$ for $q=4$. Both functions are nonmonotonous so it is possible for two different $x_{a}$ values to have the same value of $\xi^{\prime}$ and/or $\xi^{\prime \prime}$. The minimum of $\xi^{\prime}$ is located at the same $x_{a}$ as the maximum of $\xi^{\prime \prime}$, and vice versa. This is a manifestation of the Gibbs-Duhem relation

$$
\left(1-x_{2}\right)\left(\frac{\partial \mu_{1}}{\partial x_{2}}\right)_{\mathrm{n}, T}+x_{2}\left(\frac{\partial \mu_{2}}{\partial x_{2}}\right)_{\Pi, T}=0
$$

which in the present case may be rewritten as

$$
\left(1-x_{a}\right)\left(\frac{\partial \xi^{\prime}}{\partial x_{a}}\right)_{c_{a}, T}+x_{a}\left(\frac{\partial \xi^{\prime \prime}}{\partial x_{a}}\right)_{c_{a}, T}=0
$$

from which the relation between the extrema of $\xi^{\prime}$ and $\xi^{\prime \prime}$ is obvious. In the region in between the extrema the system is unstable; here we can show

$$
\left(\frac{\partial \mu_{2}}{\partial x}\right)_{\left(N_{1}+N_{2}\right) / v, T}<0
$$

from which it is clear that the system will spontaneously demix in this region. The extrema delimiting this region are the spinodal points. The dashed line in Figure 1 connects the coexisting compositions for which both $\xi^{\prime}$ and $\xi^{\prime \prime}$ are equal. In the intermediate region phase separation will occur. In Table I we give the nematic-nematic phase transition for various length ratios.

In Figure 2 we give some curves for $\xi^{\prime}$ for different values of q. (As we have seen above, the curves for $\xi^{\prime \prime}$ offer the same information.) We see that the extrema are getting less pronounced and shift toward each other for lower $q$ values. At $q=3.1672$
TABLE I: Mole Fractions and $Q, \xi$, and $\xi^{\prime \prime}$ Values at the Nematic-Nematic Phase Transition for Different Length Ratios $q$

\begin{tabular}{llccccc}
\hline$q$ & $x_{a \mid}$ & $x_{a \mid l}$ & $Q_{\mathrm{I}}$ & $Q_{\|}$ & $\xi^{\prime}$ & $\xi^{\prime \prime}$ \\
\hline 3.2 & 0.1438 & 0.3080 & 4.617 & 5.067 & -0.0566 & 4.134 \\
3.3 & 0.08739 & 0.4079 & 4.603 & 5.548 & -0.0455 & 4.300 \\
3.5 & 0.04432 & 0.5236 & 4.746 & 6.369 & -0.0295 & 4.605 \\
4 & 0.01187 & 0.6811 & 5.301 & 8.407 & -0.0101 & 5.257 \\
5 & 0.00119 & 0.8205 & 6.615 & 13.05 & -0.00115 & 6.268
\end{tabular}

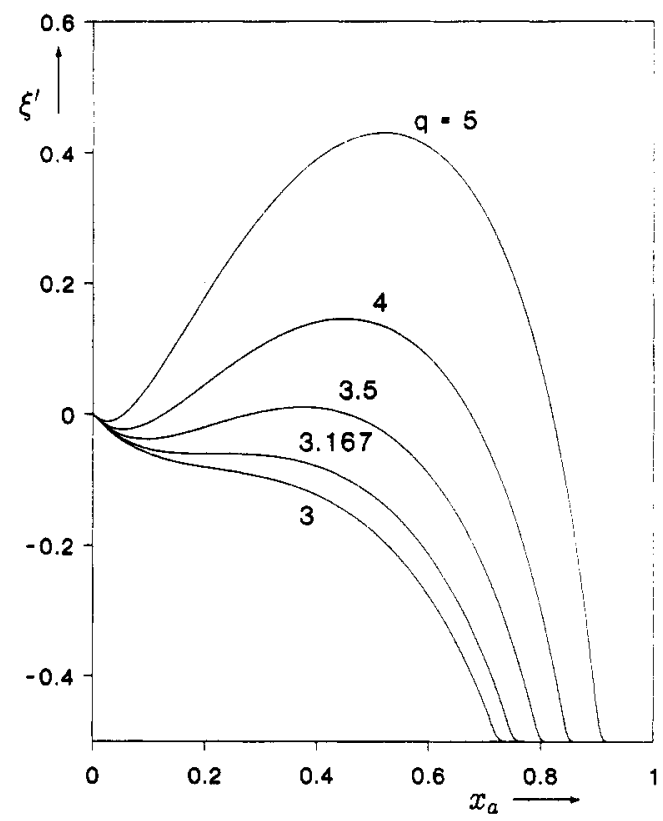

Figure 2. Mole fraction dependent part, $\xi^{\prime}$, of chemical potential $\mu_{1}$ for different values of $q$. At $q=3.167$ the extrema merge to an inflection point, so that below this $q$ value the chemical potential is decreasing monotonously and no nematic-nematic transition is possible.

they vanish, and thus below this value a nematic-nematic phase transition ceases to exist.

Finally, we want to stress that the coexistence relations discussed above are fulfilled irrespective of the concentration $c_{a}$. However, an important point we did not address is the stability of the nematic phases compared to the isotropic phase. This will be considered in the next section.

\section{Triphasic Equilibrium}

Up to now we did not take the isotropic phase into consideration. For high concentrations the free energy of this phase is dominated by the last term in eq 4 , which increases linearly with the concentration (remember that $\rho_{j k}=1$ in the isotropic phase). The same term for the nematic phase is constant since in that case $\rho_{j k} \propto c^{-1}$ (as follows from eq 9 and the discussion following eq 13). Therefore, at high concentrations, the free energy of the isotropic phase will be higher than for the nematic phase, and the isotropic phase will not interfere with the nematic-nematic phase equilibrium discussed in the preceding section.

Of course, at very low concentrations the isotropic phase is the stable state so there should be a point where the three phases are in equilibrium. This point is found by equating the osmotic pressure and chemical potentials found for the nematic-nematic equilibrium to those of the isotropic state. As will become evident later on, we may use the simplified forms valid for $x_{i} q \ll 1^{10}$ in the isotropic phase. Under this assumption equating eqs 14 and 17 gives

$$
c_{i}+c_{i}^{2}=3 c_{a}
$$

while equating the difference between eqs 16 and 15 to the 
TABLE II: Concentrations and Mole Fractions at the Isotropic-Nematic-Nematic Triple Point for Different Length Ratios $q$

\begin{tabular}{lccccc}
\hline$q$ & $c_{i}$ & $x_{i}$ & $c_{a 1}=c_{a \mid 1}$ & \multicolumn{1}{c}{$x_{a 1}$} & $x_{a \mid 1}$ \\
\hline 3.2 & 3.375 & $2.35 \times 10^{5}$ & 4.921 & 0.1438 & 0.3080 \\
3.3 & 3.390 & $1.30 \times 10^{5}$ & 4.960 & 0.08739 & 0.4079 \\
3.5 & 3.412 & $4.02 \times 10^{5}$ & 5.017 & 0.04432 & 0.5236 \\
4 & 3.438 & $2.13 \times 10^{7}$ & 5.086 & 0.01187 & 0.6811 \\
5 & 3.450 & $5.4 \times 10^{-10}$ & 5.118 & 0.001188 & 0.8205
\end{tabular}

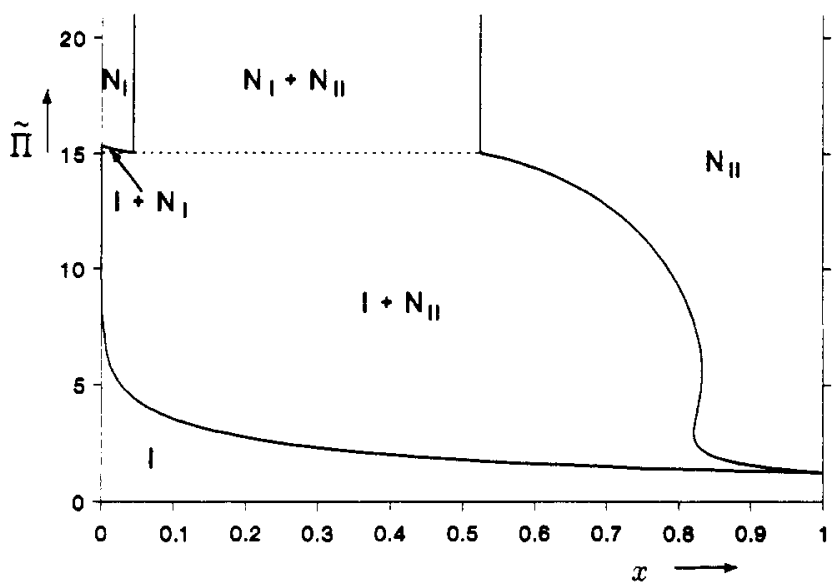

Figure 3. A $\tilde{\Pi}-x$ diagram for $q=3.5$. The dashed line is the triple line, where an isotropic (I) and two nematic $\left(N_{1}\right.$ and $\left.N_{11}\right)$ phases coexist. In case $x_{i} q$ not $\ll 1$ the full Gaussian expressions for $\mu_{1}, \mu_{2}$, and $\Pi$ were solved iteratively. Note the reentrant phenomenon ${ }^{10}$ near $x_{a}=0.82$.

difference between eqs 19 and 18 gives

$$
\ln x_{i}+2 c_{i}(q-1)=\xi^{\prime \prime}\left(x_{a}\right)-\xi^{\prime}\left(x_{a}\right)
$$

Finally, equating eqs 15 and 18 and eliminating $c_{a}$ by means of eq 28 leads to

$$
\ln c_{i}+2 c_{i}=3 \ln \frac{c_{i}+c_{i}^{2}}{3}+\ln \frac{4}{\pi}+3+\xi^{\prime}\left(x_{a}\right)
$$

Now solution of these equations is simple: $\xi^{\prime}\left(x_{a}\right)$ is known from the previous paragraph, so (30) only contains $c_{i}$ as an unknown variable, which may be solved iteratively. Then $x_{i}$ is found from (29) and $c_{a}$ is found from (28). The result for different $q$ values is given in Table II. From the very small $x_{i}$ values it is clear that indeed $x_{i} q \ll 1$ so that the use of the simplified forms of the coexistence equations is entirely justified.

To present a graphic illustration of the results obtained for $q$ $=3.5$, we give a $\bar{\Pi}-x$ diagram in Figure 3 and a diagram in terms of volume fractions in Figure 4. The volume fraction representation may be more convenient from an experimental standpoint. The volume fractions are obtained straightforwardly from the definition of $x\left(\equiv N_{2} /\left(N_{1}+N_{2}\right)\right)$ and $c$ (eq S)

$$
\begin{gathered}
\phi_{1}=\frac{\pi}{4} L_{1} D^{2} \frac{N_{1}}{V}=\frac{D}{L_{1}}(1-x) c \\
\phi_{2}=\frac{\pi}{4} L_{2} D^{2} \frac{N_{2}}{V}=\frac{D}{L_{1}} x q c
\end{gathered}
$$

Note that the three coexisting phases that lie on a triple line in the field-density presentation of Figure 3 form a three-phase triangle in the density-density representation of Figure 4. As we have seen, the mole fractions of the coexisting nematic phases are independent of concentration, which results in vertical phase boundaries in Figure 3 and corresponding straight lines in Figure 4. The tie lines which are horizontal lines in Figure 3 because of equality of osmotic pressure are denoted in Figure 4 by thin

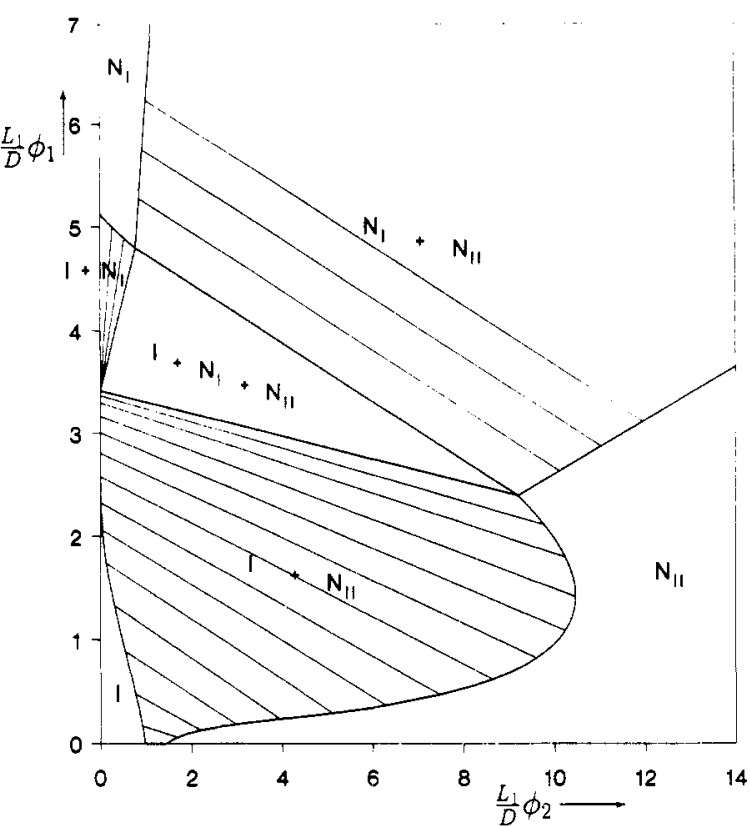

Figure 4. A phase diagram for $q=3.5$ in terms of volume fractions $\phi_{1}$ and $\phi_{2}$. To avoid the need to fix aspect ratio $L_{1} / D$, both volume fractions were multiplied by this number. Thick lines indicate phase boundaries; thin lines represent tie lines connecting coexisting phases.

lines. In a volume fraction representation these tie lines may be shown to form straight lines. ${ }^{19}$

\section{Discussion}

Our calculations based upon the Gaussian approximation give a surprisingly simple picture, of the nematic-nematic phase transition. Its origin is different from the isotropic-nematic transition, which is a result of a competition between orientationalentropy and excluded-volume terms in the free energy. In the Gaussian approximation the excluded-volume term in the nematic phase is independent of mole fraction and concentration. As a consequence, excluded volume does not play a role in the nematicnematic phase transition and the transition must be explained solely from an intricate interplay between orientational entropy and entropy of mixing. Above $q=3.167$ this leads to the existence of two nematic phases.

We wish to indicate that the present calculations are subject to some reservations. As shown in ref 8 , there exists an exact relationship between the orientational distribution functions of different rods in a polydisperse mixture, which is not fulfilled for Gaussian distribution functions. However, we wish to point out that the procedure of minimizing the free energy independently with respect to both distributions makes the value for the free energy less sensitive to the precise form of the distribution functions. The fact that our calculation does not show a critical point which closes the nematic-nematic coexistence area in the phase diagram at higher concentrations may be due to this shortcoming. This behavior was found both within the Flory theory ${ }^{11}$ and in the series solution to the Onsager theory. ${ }^{18}$

For comparison with experimental results the representation of Figure 4 is most suitable. In this figure experiments which probe the phase behavior of bidisperse mixtures by adding or evaporating the solvent, will be represented by straight lines radiating from the origin. We see from the figure that even for a low length ratio of 3.5 a large range of compositions will pass through the three-phase area. Particularly, it appears that a very small mole fraction of long rods in the isotropic phase already leads to a three-phase equilibrium upon increase of the overall concentration. Further, it is clear that -although the total number density of rods is the same in two coexisting nematic phases-the total volume fraction may be considerably different because of the difference in volume between the two kinds of rods. 
For experimental results on isotropic-nematic-nematic equilibria currently available in the literature, our theory is not quantitatively applicable because most macromolecules are not completely rigid but semiflexible, which may have considerable influence. ${ }^{5.6,8}$ This is the case for schizophyllan, where a triphasic equilibrium was found ${ }^{15,16}$ at $L_{2} / L_{1}=12.2$ but none at $L_{2} / L_{1}=$ 6.1. This suggests that for semiflexible chains a much larger length ratio is required to obtain triphasic equilibria, as might be expected. For imogolite it was found ${ }^{17}$ that a small amount of high molecular weight compound (possibly longer rods) was able to induce a triphasic equilibrium. We hope that experiments with true hard rigid rods of controlled length will be feasible in future.

\section{References and Notes}

(1) Onsager, L. Ann. N.Y. Acad. Sci. 1949, 51, 627.

(2) Onsager, L. Phys. Rev. 1942, 62, 558.

(3) Straley, J. P. Mol. Cryst. Liq. Cryst. 1973, 22, 333.
(4) Khokhlov. A. R.: Semenov, A. N. J. Stat. Phys. 1985, 38, 161

(5) Odijk, T. Macromolecules 1986, 19, 2313.

(6) Semenov, A. N.; Khokhlov, A. R. Usp. Fiz. Nauk 1988, 156, 427 [Sov. Phys. Usp. 1988, 31, 988].

(7) Khokhlov, A. R. In Liquid Crystallinity in Polymers; Ciferri, A. Ed.; VCH: New York, 1991. 1241 .

(8) Vroege, G. J.; Lekkerkerker, H. N. W. Rep. Prog. Phys, 1992, 55,

(9) Lekkerkerker, H. N. W.; Coulon, P.; van der Haegen, R.; Deblieck. R. J. Chem. Phys. 1984, 80, 3427.

(10) Odijk, T.; Lekkerkerker, H. N. W. J. Phys. Chem. 1985, 89, 2090.

(11) Flory, P. J.; Abe, A. Macromolecules 1978, $11,1119$.

(12) Flory, P. J. Proc. R. Soc. London, A 1956, 234, 60, 73

(13) Flory, P. J. Adv. Polym. Sci. 1984, 59, 1.

(14) Abe, A.; Ballauff, M. In Liquid Crystallinity in Polymers; Ciferri, A., Ed.; VCH: New York, 1991.

(15) Itou. T.; Teramoto. A. Macromolecules 1984, 17, 1419.

(16) Itou, T.; Teramoto, A. Polym. J. 1984, 16, 779.

(17) Kajiwara, K.; Donkai, N.: Hiragi, Y.; Inagaki, H. Makromol. Chem. 1986, 187,2883

(18) Birshtein, T. M.; Kolegov, B. 1.; Pryamitsyn, V. A. Vysokomol. Soyed. A 1988, 30, 348 [Polym. Sci. U.S.S.R. 1988, 30, 316],

(19) Bartlett, P. J. Phys.: Condens. Matter 1990, 2, 4979. 